\title{
Effect of Rehabilitation Intervention on Severe Pneumonia in Children
}

\author{
G. ZHU, J. ZHANG ${ }^{1}$, G. TONG ${ }^{2 *}$
}

Outpatient Nursing, Chun'an First People's Hospital, No.1869 Huanhu Road, Qiandaohu Town, Chun'an, Hangzhou City, Zhejiang Province 311700, 1Hospital Office, Hangzhou First People's Hospital, No.261 Huanshan Road, Hangzhou City, Zhejiang Province 310006, 2Department of Nursing, Chun'an Chinese Medicine Hospital, No. 1 Xin'an West Road, Qiandaohu Town, Chun'an, Zhejiang Province 311700, China

Zhu et al.: Rehabilitation intervention on children with severe pneumonia

\begin{abstract}
In order to study the effect of rehabilitation intervention on children with severe pneumonia, this study used 48 cases of severe pneumonia in children as the research object. The children were divided into 2 groups according to the random principle, 24 people in each group, one group was experimental group (irrigation group), Bronchoalveolar irrigation rehabilitation and conventional anti-infective treatment were performed. The other group was the control group (routine group) and only the same anti-infective treatment was performed. The degree of rehabilitation of the children was determined by the $\mathrm{C}$-reactive protein, procalcitonin and the imaging inflammatory absorption area before and after treatment in the two groups of patients. The study found that the $\mathrm{C}$-reactive protein and procalcitonin abnormalities in the experimental group were lower than those in the control group, and the imaging inflammatory absorption area was significantly larger than that in the control group. This shows that bronchoalveolar irrigation rehabilitative treatment can increase the recovery rate of child patient, the treatment effect is far better than the children who only carry out conventional anti-infection, also shows that bronchoalveolar irrigation rehabilitation treatment has excellent clinical treatment effect.
\end{abstract}

Key words: Severe pneumonia, pediatric, rehabilitation treatment

Pneumonia, an infection caused by various pathogens or caused by other factors, leading to acute inflammation of the lung parenchyma and/or interstitial parts, can lead to different degrees of hypoxia and infective intoxication symptoms. One of the leading causes of death among children under five is pneumonia. Pneumonia is also one of the major diseases in childhood in China ${ }^{[1]}$. About 21 million children in China suffer from pneumonia every year and $7 \%$ to $13 \%$ of them are severe pneumonia. Severe pneumonia is a common emergency respiratory disease in children ${ }^{[2]}$. It is acute and has a strong onset. It often affects the whole body. Typical clinical manifestations and signs often fail to attract attention or be concealed in clinical work, especially in infants and young children. Most of child patient died of various complications of severe pneumonia. Pediatric pneumonia is a group of respiratory diseases caused by bacterial, viral and other foreign bodies ${ }^{[3]}$. In recent years, about half of children in intensive care units are cases of severe pneumonia in children. Some patients may continue to develop severe pneumonia because not be timely treated due to mild case, and some application of antibiotics is unreasonable, as well as self-resistance and other reasons. In addition to severe respiratory manifestations, some cases may have significant central nervous system involvement and other systemic symptoms ${ }^{[4,5]}$. Pneumonia in children are extremely easy to progress rapidly, from local lung infection to systemic infection, respiratory failure, heart failure, toxic encephalopathy, microcirculatory disturbance, shock, disseminated intravascular coagulation and multiple organ failure. It is a serious threat to children's life safety. A large proportion of children with severe pneumonia, even if they save their lives, will leave longterm cough, wheezing, chest radioactivity prolonged, limited activity and other sequelae ${ }^{[6,7]}$. Children with severe pneumonia are found early in clinical practice. Early diagnosis and timely treatment are of great significance in saving the lives of child patient.

*Address for correspondence 
In recent years, with the rapid development of scientific instruments and equipment such as electronic instruments and optical fibers, bronchoscopy suitable for children has been used as a skill operation tool for children's respiratory doctors ${ }^{[8,9]}$. It is gradually used in pediatric clinical practice and it can accurately arrive at pulmonary location of lesion, and conduct local sampling, culture, finding pathogens, which is the characteristic of bronchoalveolar irrigation treatment. Therefore, it has important practical and social significance to explore the clinical efficacy of bronchoalveolar irrigation in the acute phase of severe pneumonia, explore new treatment options for children with severe pneumonia.

\section{MATERIALS AND METHODS}

\section{Patients:}

In this study, 48 patients who underwent treatment in Chun'an First People's Hospital and met the diagnostic criteria for severe pneumonia were randomized into two groups, 24 patients in each group. Children in the routine group were treated with antibiotics and given conventional treatment such as symptomatic treatment. In the irrigation group, bronchoalveolar irrigation was performed by bronchoscopy on the basis of conventional treatment with the consent of the patient and his or her guardian. The informed consent signed by the patients or their family members was obtained and this study was approved by the medical ethics committee of Chun'an First People's Hospital.

\section{Diagnostic criteria for severe pneumonia:}

Diagnostic criteria for community-acquired pneumonia: clinical fever (sometimes may not occur); cough, expectoration, wheezing, or aggravation of the original symptoms, cough and sputum, some cases may have chest pain; some cases of lungs auscultation of audible wet rale in certain area; or signs of lung consolidation; chest imaging examinations may have large, patchy infiltration shadows or interstitial changes, some may be associated with pleural effusion; blood routine examination shows the number of white blood cells $>10 \times 10^{9} /$ Lor $<4 \times 10^{9} \mathrm{~L}$, or the phenomenon of nuclear left shift; at the same time, it needs to exclude tuberculosis, non-infectious interstitial lung disease, lung cancer, atelectasis, pulmonary vasculitis, pulmonary edema, pulmonary eosinophilic infiltration, etc. The diagnostic criteria for severe pneumonia are based on pneumonia: poor general condition; apastia or dehydration; disturbance of consciousness; increased respiratory rate; cyanosis; difficulty breathing (groan, nares flaring, three concave sign); multiple lung involvement or $\geq 2 / 3$ of lung infiltration; pleural effusion; saturation of pulse oximetry $\leq 0.92$; extra-pulmonary complications, any of the above can diagnose severe pneumonia.

\section{Bronchoalveolar irrigation treatment:}

Preoperative anesthesia: a combination of local anesthesia of the respiratory tract and general anesthesia with intravenous administration. $2 \%$ lidocaine (2-4 $\mathrm{mL}$ ) was inhaled $15 \mathrm{~min}$ before surgery; penehyclidine hydrochloride $(0.02 \mathrm{mg} / \mathrm{kg})$ was intravenously injected by anesthesiologist $10 \mathrm{~min}$ before surgery; ketamine (2 $\mathrm{mg} / \mathrm{kg}$ ) was given intravenously $5 \mathrm{~min}$ before surgery; propofol $(6-8 \mu \mathrm{g} / \mathrm{kg} / \mathrm{h})$ may be administered continuously or midazolam $(0.1 \mathrm{mg} / \mathrm{kg})$ may be given intravenously depending on the case.

Intraoperative operation: the child was placed in the supine position. Under the conventional intravenous anesthesia, the bronchoscope was gently sent through the nasal cavity, and was entered into weasand through the glottis. In the first place observe the left and right main bronchus, the lung lobes, the color and congestion of bronchial mucosa at lung segment; size, shape, abnormal secretion and occlusion of the bronchus. Secondly, the bronchoalveolar irrigation fluid was taken under bronchoscopy and tested in time. Then perform repeated irrigation treatment by injecting the irrigation fluid into the biopsy channel. The irrigation solution was prepared according to a $1: 1$ ratio of $0.9 \%$ sodium chloride solution plus metronidazole solution, and the irrigation volume was $5-10 \mathrm{~mL}$ each time; the total irrigation volume was $10 \mathrm{~mL} / \mathrm{kg}$. The child's vital signs such as breathe, heart rate, blood pressure, blood oxygen saturation and other should be observed during the operation, if the child's vital signs are not stable during the operation, and there is manifestation of lip cramps, increased heart rate, decreased blood oxygenation, the child should be immediately relieved of airway obstruction and keep the upper airway unobstructed, pressurize oxygen should be given, and ensure the oxygen supply of the child. If there are persistent symptoms, the operation should be terminated immediately, and the first aid procedure should be entered.

Postoperative observation: After the end of bronchoalveolar irrigation treatment, observe the vital signs and cough of the child, be alert to laryngospasm and cough, and the monitoring time is about 3-5 $\mathrm{h}$. After 2-4 h of irrigation treatment, if the child's vital 
signs are stable, no changes in the condition such as laryngospasm and cough, then the child can drink water; if no abnormalities later, fluid food can be given.

\section{Specimen collection method:}

Blood culture collection method: Collect $3 \mathrm{ml}$ of blood on the morning of the child with severe pneumonia after admission to the hospital with an empty belly before anti-infective treatment, and the medium was sent for examination.

Collection method of serum virus antibody and mycoplasma antibody: In children with severe pneumonia, $3 \mathrm{ml}$ of blood was collected on the $8^{\text {th }} \mathrm{d}$ of the total disease course with an empty belly, and inject the blood into separated vacuum blood collection tube containing the coagulant, and the serum was separated and sent for examination.

Bronchoalveolar irrigation fluid collection method: In children with severe pneumonia undergoing bronchoalveolar irrigation treatment, in the case of protective sampling, the bronchoscope is placed at the nozzle where the lesion is located, and the sputum collector is connected. Slowly inject $10 \mathrm{ml}$ of normal saline, open the aspirator, attract the bronchial and lung fluid into the sputum collector, and send it for inspection.

\section{Laboratory testing methods:}

Detection method of procalcitonin: use automatic immunoassay analyzer and original kit. The reference range of procalcitonin is $0-0.1 \mathrm{ng} / \mathrm{mL}$, and $>0.1 \mathrm{ng} / \mathrm{mL}$ is abnormal;

The detection method of blood C-reactive protein: using Roche automatic biochemical analyzer and DiaSys Diagnostic C-reactive protein assay kit for detection, the reference range of C-reactive protein is $0-10 \mathrm{mg} / \mathrm{L}$, $>10 \mathrm{mg} / \mathrm{L}$ is abnormal.

\section{Imaging criteria for determining clinical efficacy:}

\section{After postoperative imaging examination}

Recovery: The child's body temperature gets to normal, cough and lung rale and other symptoms disappeared, chest x-ray or chest computed tomography (CT) showed inflammation absorption $>75 \%$.

Effective: the child's body temperature gets to normal, cough and lung rale and other symptoms are relieved, chest $\mathrm{x}$-ray or chest CT suggests inflammation absorption $>50 \%$.
Invalid: body temperature $>38^{\circ}$, cough and lung wet rale and other symptoms are not alleviated, chest X-ray or chest CT showed inflammation is not absorbed or enlarged.

\section{Statistical methods:}

Statistical analysis was performed using Statistical Package for the Social Sciences (SPSS) 24.0 statistical software. The count data were descriptive statistical methods, expressed as percentage and composition ratio, and the count data conforming to the normal distribution. The chi-square test was used, $\mathrm{p}<0.05$, which was statistically significant. The measured data of normal distribution were statistically significant by t-test, mean \pm standard deviation $(\mathrm{x} \pm \mathrm{s}), \mathrm{p}<0.05$.

\section{RESULTS AND DISCUSSION}

A total of 48 subjects were included in the study, including 16 males $(33.3 \%)$ and 32 females $(66.7 \%)$. The age of onset was $0-12$ y old, with an average age of $6.12 \mathrm{y}$. The age distribution was as shown in fig. 1. The comparison of gender and mean age between the routine group and the irrigation group shows $\mathrm{p}>0.05$, the difference was not significant, and it was not statistically significant and comparable.

Among the 48 children with severe pneumonia who met the inclusion criteria, 28 patients had complications, accounting for $58.3 \%$; 20 patients had no complications, accounting for $41.7 \%$. A total of 65 complications occurred, and the results are shown in fig. 2a. It can be observed that the incidence of pleurisy is the highest in the complications of severe pneumonia. The complications and comparison between the routine group and the irrigation group are shown in fig. $2 \mathrm{~b}$. Compared with the routine group, the complication rate of the irrigation group was higher than that of the routine group, $\mathrm{p}>0.05$, the difference was not

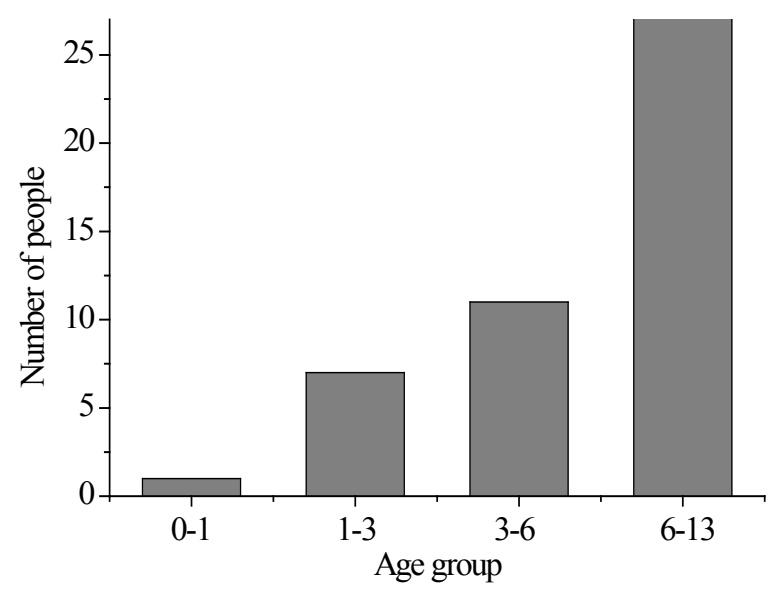

Fig. 1: Age distribution of children with severe pneumonia 
statistically significant and comparable.

In 48 patients, imaging showed 35 cases of unilateral lung involvement, accounting for $72.9 \%$, and bilateral lung involvement in 13 cases, accounting for $27.1 \%$. Of the 35 patients with unilateral lung involvement, 22 were in the right lung, accounting for $62.9 \%$, and 13 in the left lung, accounting for $37.1 \%$. The results are shown in fig. $3 \mathrm{a}$ and $3 \mathrm{~b}$. The imaging findings of the

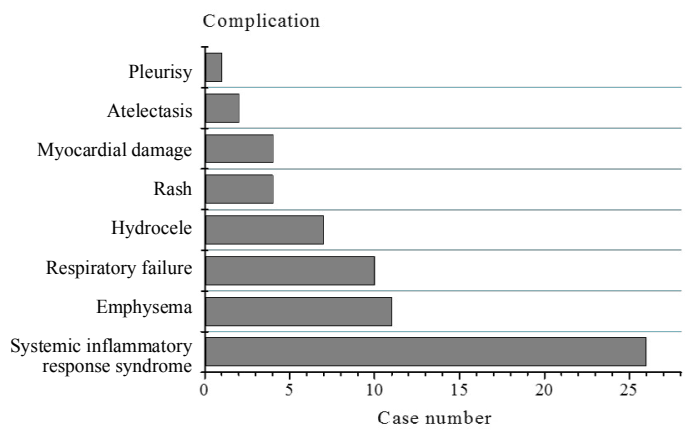

A

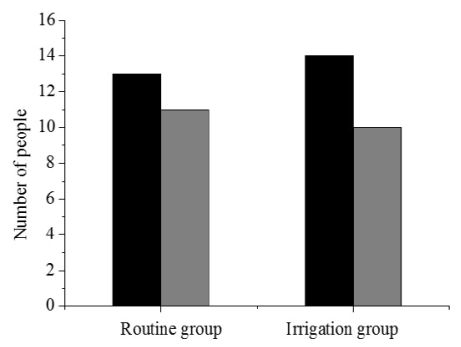

Fig. 2: Complications in patients

A. Type and incidence of complications in children, $B$. Comparison of the number of complications in the two groups lungs involvement in the two groups were compared and the results are shown in fig. 3c. The results showed that the incidence of bilateral lung involvement in the irrigation group was lower than that in the routine group $(\mathrm{p}<0.05)$. The difference was statistically significant.

Among the 48 children with severe pneumonia who met the inclusion criteria, there are 45 cases of abnormal blood C-reactive protein, accounting for $93.8 \%, 3$ cases of normal, accounting for $6.3 \%$; 34 cases of abnormal procalcitonin, accounting for $70.8 \%, 14$ cases of normal, accounting for $29.2 \%$, the results are shown in fig. $4 \mathrm{a}$ and $4 \mathrm{~b}$. Comparing the $\mathrm{C}$-reactive protein and the procalcitonin of two groups, the abnormal rate of C-reactive protein in the irrigation group was lower than that in the routine group $(\mathrm{p}<0.05)$. The difference was statistically significant, as shown in fig. 4c. After treatment, the abnormal rate of procalcitonin in the irrigation group was lower than that in the routine group, $\mathrm{p}<0.05$, and the difference was statistically significant, as shown in fig. $4 \mathrm{~d}$.

48 children with severe pneumonia who met the inclusion criteria were treated. When the clinical symptoms were completely relieved for $5 \mathrm{~d}$ and the clinical discharge criteria were met, imaging examination is needed to observe the absorption area. For cases that imaging absorption area $>75 \%, 9$ cases in the routine group was, accounting for $37.5 \%, 14$ cases in irrigation group, accounting for $58.3 \%$; imaging absorption area $>50 \%, 3$ cases in routine group, accounting for $12.5 \%$, 7 cases in irrigation group, accounting for $29.2 \%$; no

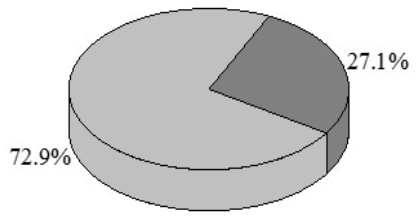

A

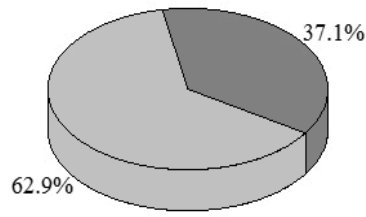

B

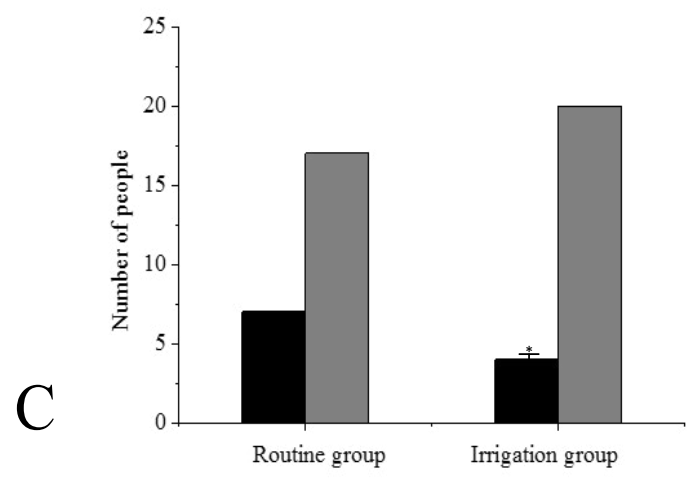

Fig. 3: Imaging of lung involvement $\left({ }^{*} p<0.05\right)$

A: Proportion of unilateral and bilateral lung involvement; B: Proportion of left and right lung involvement; C: Comparison of lung involvement in both groups 
A
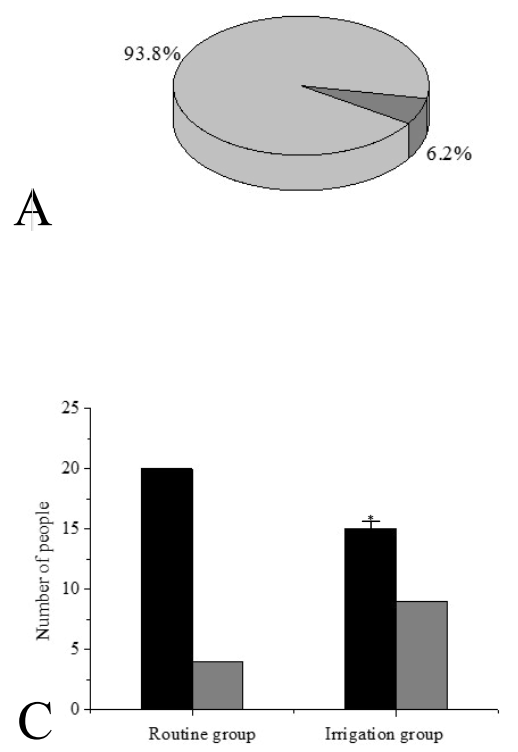

B
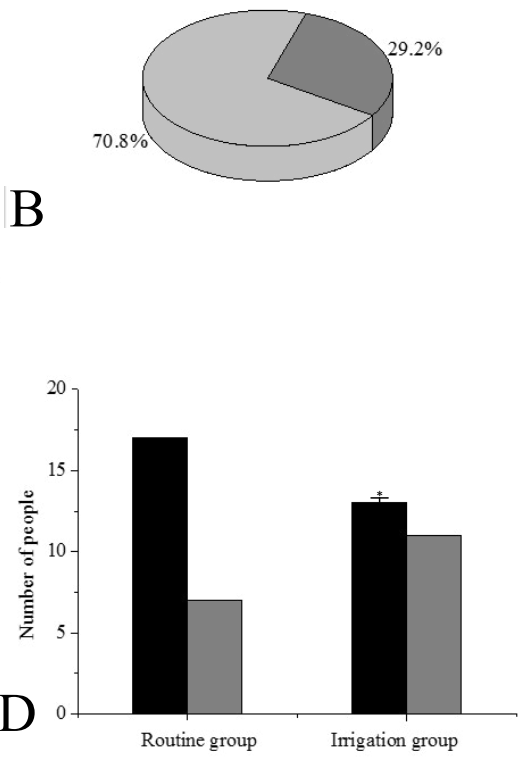

Fig. 4: Abnormalities of blood $\mathrm{C}$-reactive protein and procalcitonin $(* \mathrm{p}<0.05)$

A: Abnormal blood C-reactive protein; B: Abnormal blood procalcitonin; C: Abnormalities of blood C-reactive protein in the two groups; D: Abnormalities of procalcitonin in the two groups

absorption or area enlarged, 12 cases in routine group $50.0 \%, 3$ cases in the irrigation group, accounting for $12.5 \%$. The comparison result of the imaging absorption area of the two groups is shown in fig. 5. The results showed that when the patient met the clinical discharge standard, the imaging absorption area of the irrigation group was higher than that of the routine group, $p<0.05$, and the difference was statistically significant.

For the 48 children who met the inclusion criteria, the clinical efficacy of the routine group and the irrigation group was observed, and the clinical recovery was achieved. Clinical recovery: 6 cases in the routine group, accounting for $25.0 \%$, and 11 cases in the irrigation group, accounting for $45.8 \%$. Clinically effective: 5 cases in the routine group, accounting for $20.1 \%, 10$ cases in the irrigation group, accounting for $41.7 \%$. Invalid: 13 cases in the routine group, accounting for $54.2 \%, 3$ cases in the irrigation group, accounting for $12.5 \%$. The comparison result of the recovery rates between the two groups is shown in fig. 6 .

Among the 48 children with severe pneumonia in this study, female is slightly more than male. The age of onset was 0-12 y old, with an average age of $6.12 \mathrm{y}$. Some patients had complications before treatment and the main complication was pleurisy. The children were mainly affected by unilateral lung involvement, and the unilateral lung involvement was mainly affected by right lung involvement, which was improved after treatment. The results of this study showed that after bronchoalveolar irrigation rehabilitation, the situation of severe pneumonia in children was significantly

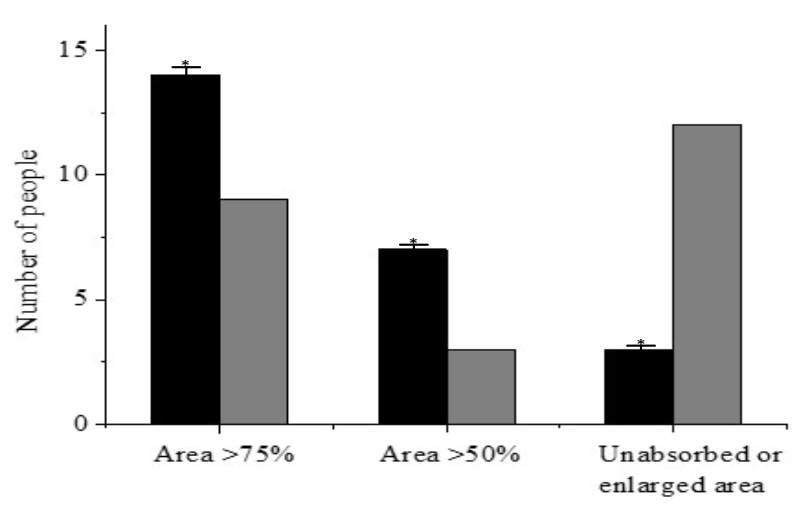

Fig. 5: Comparison of imaging improvement between the two groups $(* \mathbf{p}<0.05)$

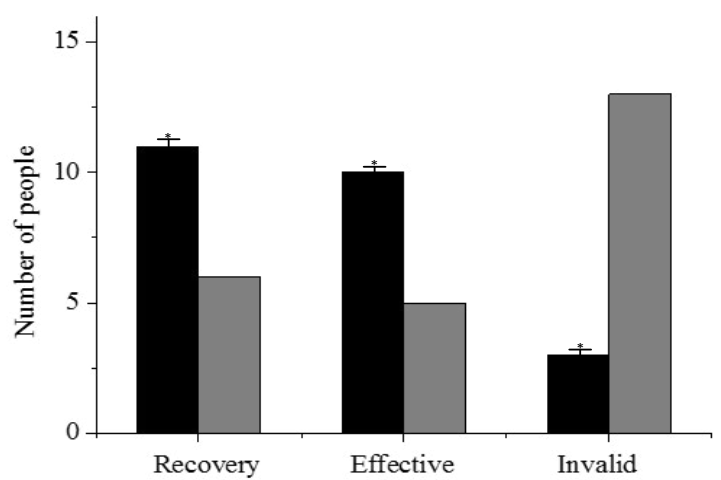

Fig. 6: Comparison of clinical efficacy between the two groups $(* \mathbf{p}<0.05)$

improved compared with conventional medication, mainly reflected in the increase of imaging inflammatory absorption area, and the clinical recovery rate was significantly increased. This shows that the efficacy of bronchoalveolar irrigation rehabilitation is much higher than that of conventional anti-infective medicine. It also 
shows that bronchoalveolar irrigation rehabilitation has a very good therapeutic effect on children with severe pneumonia.

\section{Acknowledgements:}

None

\section{Conflict of Interests:}

The authors declared no conflict of interest.

\section{REFERENCES}

1. Wang K, Gao M, Yang M, Meng F, Li D, Lu R, et al. Transcriptome analysis of bronchoalveolar lavage fluid from children with severe Mycoplasma pneumoniae pneumonia reveals novel gene expression and immunodeficiency. Hum Genomics 2017;11(1):4.

2. Vitali I, Genovese O, Conti G, Raffaelli L, D'Addona A, Dell'Anna A, et al. Effectiveness of a ventilator-associated pneumonia prevention bundle in critically ill children. J Biol Regul Homeost Agents 2019;33(2):643-7.

3. Shi Z, Qin Y, Zhu Y, Pan X, Zhou X, Tan Y, et al. Effect of bronchoalveolar lavage with fiberoptic bronchoscopy combined with vibration sputum drainage on mechanically ventilated patients with severe pneumonia: a prospective randomized controlled trial in 286 patients. Zhonghua Wei Zhong Bing Ji Jiu Yi Xue 2017;29(1):66-70.

4. Wang Z, Liu Q, Wang X, Wang Y, Zhang J, Zhou W, et al. Expressions of microRNA-127-5p in bronchoalveolar lavage fluid of patients with severe pneumonia and its diagnostic value. Zhonghua Wei Zhong Bing Ji Jiu Yi Xue 2017;29(7):592-5.
5. Nakagome K, Shoda H, Shirai T, Nishihara F, Soma T, Uchida $\mathrm{Y}$, et al. Eosinophil transendothelial migration induced by the bronchoalveolar lavage fluid of acute eosinophilic pneumonia. Respirology 2017;22(5):913-21.

6. Demir S, Saison J, Senechal A, Mornex JF. A severe Mycoplasma pneumoniae pneumonia inducing an acute antibody-mediated pulmonary graft rejection. Lung India 2017;34(1):85-7.

7. Gao M, Wang $\mathrm{K}$, Yang M, Meng $\mathrm{F}$, Lu R, Zhuang $\mathrm{H}$, et al. Transcriptome analysis of Bronchoalveolar lavage fluid from children with mycoplasma pneumoniae pneumonia reveals natural killer and T cell-proliferation responses. Front Immunol 2018;9:1403.

8. Tatsumi H, Yamamoto M, Yasui K, Miyake T. Clinical significance of cognitive rehabilitation and psychoeducational intervention for family caregivers of patients with posterior cortical atrophy: a longitudinal study. Psychogeriatrics 2018;18(1):77-8.

9. Lo WL, Mao YR, Li L, Lin AH, Zhao JL, Chen L, et al. Prospective clinical study of rehabilitation interventions with multisensory interactive training in patients with cerebral infarction: study protocol for a randomised controlled trial. Trials 2017;18(1):173.

This is an open access article distributed under the terms of the Creative Commons Attribution-NonCommercial-ShareAlike 3.0 License, which allows others to remix, tweak, and build upon the work non-commercially, as long as the author is credited and the new creations are licensed under the identical terms

This article was originally published in a special issue, "Trends in Therapeutic Management of Various Clinical Conditions II" Indian J Pharm Sci 2021:83(2)Spl issue;18-23 